\title{
Big data clustering techniques based on Spark: a literature review
}

\author{
Mozamel M. Saeed ${ }^{\text {Corresp., } 1}$, Zaher Al Aghbari ${ }^{2}$, Mohammed Alsharidah ${ }^{1}$ \\ ${ }^{1}$ Department of Computer Science, Prince Sattam Bin Abdul Aziz, Riyadh, Saudi Arabia \\ 2 Department of Computer Science, University of Sharjah, Sharjah, United Arab Emirates \\ Corresponding Author: Mozamel M. Saeed \\ Email address: m.musa@psau.edu.sa
}

A popular unsupervised learning method, known as clustering, is extensively used in data mining, machine learning and pattern recognition. The procedure involves grouping of single and distinct points in a group in such a way that they are either similar to each other or dissimilar to points of other clusters. Traditional clustering methods are greatly challenged by the recent massive growth of data. Therefore, several research works proposed novel designs for clustering methods that leverage the benefits of Big Data platforms, such as Apache Spark, which is designed for fast and distributed massive data processing. However, Spark-based clustering research is still in its early days. In this systematic survey, we investigate the existing Spark-based clustering methods in terms of their support to the characteristics Big Data. Moreover, we propose a new taxonomy for the Spark-based clustering methods. . To the best of our knowledge, no survey has been conducted on Spark-based clustering of Big Data. Therefore, this survey aims to present a comprehensive summary of the previous studies in the field of Big Data clustering using Apache Spark during the span of 2010-2020. This survey also highlights the new research directions in the field of clustering massive data. 
1 Big Data Clustering Techniques based on Spark: A 2 literature review

3

4

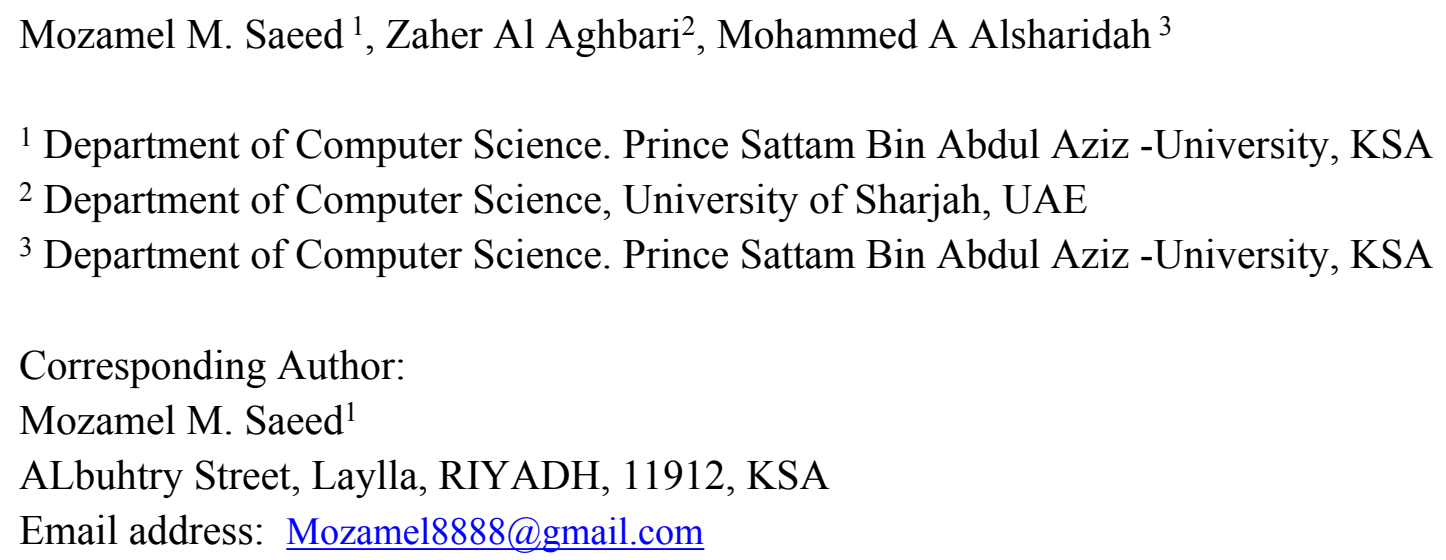

\section{Abstract}

A popular unsupervised learning method, known as clustering, is extensively used in data mining, machine learning and pattern recognition. The procedure involves grouping of single and distinct points in a group in such a way that they are either similar to each other or dissimilar to points of other clusters. Traditional clustering methods are greatly challenged by the recent massive growth of data. Therefore, several research works proposed novel designs for clustering methods that leverage the benefits of Big Data platforms, such as Apache Spark, which is designed for fast and distributed massive data processing. However, Spark-based clustering research is still in its early days. In this survey, we investigate the existing Spark-based clustering methods in terms of their support to the characteristics Big Data. Moreover, we propose a new taxonomy for the Spark-based clustering methods. . To the best of our knowledge, no survey has been conducted on Spark-based clustering of Big Data. Therefore, this survey aims to present a comprehensive summary of the previous studies in the field of Big Data clustering using Apache Spark during the 
31 span of 2010-2020. This survey also highlights the new research directions in the field of clustering

32 massive data.

33

34 Keywords Spark-based clustering; Big Data clustering; Spark; Big Data

35

36

37

38

39

40

41

42

43

44

45

46

47

48

49

50

51

52

53

54

55

\section{Introduction}

With the emergence of 5G Technologies, a tremendous amount of data is being generated very quickly, which turned into a massive amount that is termed as Big Data. The attributes of Big Data such as huge volume, a diverse variety of data, high velocity and multivalued data makes data analytics difficult. Moreover, extracting meaningful information from such volumes of data is not an easy task [1]. As an indispensable tool of data mining, clustering algorithms play an essential role in big data analysis. Clustering methods are mainly divided into density-based, partitionbased, hierarchical, and model-based clustering.

7 All these clustering methods are developed to tackle the same problems of grouping single and distinct points in a group in such a way that they are either similar to each other or dissimilar to points of other clusters. They work as follows: 1) randomly select initial clusters and 2) iteratively optimize the clusters until an optimal solution is reached [2]. Clustering has an enormous application. For instance, clustering is used in intrusion detection system for the detection of anomaly behaviours [3],[4]. Clustering is also used extensively in text analysis to classify documents into different categories [5],[6]. However, as the scale of the data generated by modern technologies is rising exponentially, these methods become computationally expensive and do not scale up to very large datasets. Thus, they are unable to meet the current demand of contemporary 
56 data-intensive applications [7]. To handle big data, clustering algorithms must be able to extract

57 patterns from data that are unstructured, massive and heterogeneous.

58 Apache Spark is an open-source platform designed for fast-distributed big data processing.

59 Primarily, Spark refers to a parallel computing architecture that offers several advanced services

60 such machine learning algorithms and real time stream processing [8]. As such, Spark is gaining

61 new momentum, a trend that has seen the onset of wide adoption by enterprises because of its

62 relative advantages. Spark grabbed the attention of researchers for processing big data because of

63 its supremacy over other frameworks like Hadoop MapReduce [9]. Spark can also run in Hadoop

64 clusters and access any Hadoop data source. Moreover, Spark Parallelization of clustering

65 algorithms is an active research problem, and researchers are finding ways for improving the

66 performance of clustering algorithms. The implementation of clustering algorithms using spark has

67 recently attracted a lot of research interests.

68 This survey presents the state-of-the-art research on clustering algorithms using Spark Platform.

69 Research on this topic is relatively new. Efforts started to increase in the last few years, after the

70 Big Data platform, such as Apache Spark, was developed. This resulted in a number of research

71 works that designed clustering algorithms to take advantage of the Big Data platforms, specially

72 Spark due to its speed advantage. Therefore, review articles are needed to show an overview of the

73 methodologies of clustering Big Data, highlight the findings of research and find the existing gaps

74 this area.

75 Consequently, few researchers have published review articles [10 - 20]. These review articles

76 are either before 2016 or do not present a comprehensive discussion on all types of clustering

77 methods. Therefore, a comprehensive review on clustering algorithms of big data using Apache

78 Spark is needed because it is conducted based on a scientific search strategy. To the best of our 
79 knowledge, no survey has been conducted on Spark-based clustering of Big Data. For this purpose,

80 this survey aims to present a comprehensive summary of the previous studies in the field of Big

81 Data clustering using Apache Spark during the span of 2010-2020. The contributions of this review

82 are:

- This review includes quality literature from pre-defined resources and based on predefined inclusion/exclusion criteria. Therefore, out of the 476 full-text articles studied, 91 articles were included.

- A taxonomy of Spark-based clustering methods that may point researchers to new techniques or new research areas.

- A comprehensive discussion on the existing Spark-based clustering methods and the research gaps in this area. Furthermore, we presented some suggestions for new research directions.

We believe that researchers in the general area of cluster Big Data and specially those designing and developing Spark-based clustering would benefit from the findings of this comprehensive review.

94 The rest of this survey is organised as follows. Section II presents the related surveys to the topic of clustering Big data. In Section III, we present a background on the Apache Spark. Section IV explains the methodology used in this survey. Section V discusses the different Spark clustering 97 algorithms. In Section VI, we present our discussion the clustering big data using Spark and future work. Finally, we conclude the paper in Section VII. 


\section{II. Background}

103

104

105

106

107

108

109

110

111

112

113

114

115

116

117

118

119

120

121

122

123 124

125

Over the last decade, a huge amount of data has been generated. This increase in data volume is attributed to the growing adoption of mobile phones, cloud-based applications, artificial Intelligence and Internet of Things. Contemporary data come from different sources with high volume, variety and velocity, which make the process of mining extremely challenging and time consuming [10]. These factors have motivated the academic and the industrial communities to develop various distributed frameworks to handle the complexity of modern datasets in a reasonable amount of time.

In this regard, Apache spark, a cluster computing, is an emerging parallel platform that is costeffective, fast, fault-tolerant and scalable. Thereby, such features make Spark an ideal platform for the dynamic nature of the contemporary applications. Spark is designed to support a wide range of workloads including batch applications, iterative algorithms, interactive queries, and streaming [11]. Spark extends the Hadoop model and support features such as in-memory computation and resilient distributed dataset, which make it significantly faster than the traditional Hadoop mapreduce for processing large data [12].

As shown in Fig. 1, at the fundamental level, spark consist of two main components; A driver which takes the user code and convert it into multiple tasks which can be distributed across the hosts, and executors to perform the required tasks in parallel. Spark is based on RDD, which is a database tables that is distributed across the nodes of the cluster. Spark supports two main operations; Transformations; and actions. Transformation preform operations on the RDD and generates new one; Action operations are performed on RDD to produce the output [13]. 


\section{A. Spark Components}

127 1) Spark Core

128 Spark core is the foundation of Apache Spark and contains important functionalities, including 129 components for task scheduling, memory management, fault recovery, interacting with storage 130 systems. Spark Core is also home to the API that defines resilient distributed datasets (RDDs), 131 which are Spark's main programming abstraction. RDDs represent a collection of items distributed 132 across many compute nodes that can be manipulated in parallel. Spark Core provides many APIs 133 for building and manipulating these collections [14].

\section{2) Spark Streaming}

135 Spark streaming component provide scalable, high throughput API for processing of real-time 136 stream data from various sources. Examples of data streams include logfiles generated by 137 production web servers, or queues of messages containing status updates of a particular system $138[15]$.

139 3) Spark MLlib

140 Spark comes with a library MLlib which supports several common Machine Learning algorithms 141 that include classification, regression, clustering, features extraction, transformation and 142 dimensionality reductions [16].

\section{4) Spark SQL}

144 Spark SQL [17] is a module for processing structured data, which also enables users to perform 145 SQL queries. This module is based on the RDD abstraction by providing Spark core engine with 146 more information about the structure of the data. 


\section{5) Spark Graphx}

150 GraphX[18] is a library for manipulating graphs (e.g., a social network's friend graph) and 151 performing graph-parallel computations. Like Spark Streaming and Spark SQL, GraphX extends

152 the Spark RDD API, allowing us to create a directed graph with arbitrary properties attached to 153 each vertex and edge. GraphX also provides various operators for manipulating graphs (e.g., 154 subgraph and mapVertices) and a library of common graph algorithms.

155

156

\section{B. Clustering Big Data}

157 Clustering is a popular unsupervised method and an essential tool for Big Data Analysis. 158 Clustering can be used either as a pre-processing step to reduce data dimensionality before running 159 the learning algorithm, or as a statistical tool to discover useful patterns within a dataset. Clustering 160 methods are based on iterative optimization [19]. Although these methods are effective in 161 extracting useful pattern from datasets, they consume massive computing resources and come with 162 high computational costs due to the high dimensionality associated with contemporary data 163 applications [20].

164

165

\section{Challenges of Clustering Big Data}

166 The challenges of clustering big data are characterized into three main components:

167 1) Volume: as the scale of the data generated by modern technologies is rising exponentially, 168 clustering methods become computationally expensive and do not scale up to very large 169 datasets. 
170 2) Velocity: this refers to the rate of speed in which data is incoming to the system. Dealing with

171 high velocity data requires the development of more dynamic clustering methods to derive 172 useful information in real time.

173 3) Variety: Current data are heterogeneous and mostly unstructured, which make the issue to 174 manage, merge and govern data extremely challenging.

175 Conventional clustering algorithms cannot handle the complexity of big data due the above 176 reasons. For example, k-means algorithm is an NP-hard, even when the number of clusters is 177 small. Consequently, scalability is a major challenge in big data. Traditional clustering methods 178 were developed to run over a single machine and various techniques are used to improve their 179 performance. For instance, sampling method is used to perform clustering on samples of the data and then generalize it to the whole dataset. This reduces the amount of memory needed to process the data but results in lower accuracy. Another technique is features reduction where the dimension of the dataset is projected into lower dimensional space to speed up the process of mining [21]. Nevertheless, the constant growth in big data volume exceeds the capacity of a single machine, which underline the need for clustering algorithms that can run in parallel across multiple machines. For this purpose, Apache spark has been widely adapted to cope with big data clustering issues. Spark provides in-memory, distributed and iterative computation, which is particularly useful for performing clustering computation. It also provides advanced local data caching system, fault-tolerant mechanism and faster-distributed file system.

\section{Literature Review}


194 this area has already come up with some surveys and taxonomies, but most of them are related to

195 Hadoop platform while others are outdated or do not cover every aspect of clustering big data 196 using Spark.

197 The work in [22] conducted a survey on Hadoop framework for big data processing. Different 198 features of Hadoop map-reduce are discussed to deal with the problems of scalability and 199 complexity for processing big data. [23] conducted a survey on k-means using map reduce model. 200 In this article the technical details of parallelizing k-means using Apache Hadoop is discussed. 201 According to this research, k-means method is regarded as a viable approach for certain 202 applications of big data clustering and has attracted many researchers than any other techniques. 203 On the other hand, [24] conducted a survey on the major challenges for big data processing using 204 Hadoop map- reduce. According to this survey, network latency is the main limitation of Hadoop.

205 The authors of [25] conducted a survey on big data and Hadoop architecture. The paper classifies 206 existing Hadoop based systems and discusses their advantages and disadvantages. The paper 207 explains the different technologies (Hbase, Hive, Pig, etc.) used with the Hadoop distributed file 208 system (HDFS). [26] conducted a survey on large scale data processing using Hadoop over the 209 cloud. The main components of Hadoop platform and their functionalities are discussed.

210 The work in [27] conducted a comprehensive survey on spark ecosystem for processing large211 scale data. In this article, spark architecture and programming model is introduced. The authors 212 discussed the pros and cos of spark platform as well as the various optimization techniques used 213 for improving spark performance for processing large scale data.

214 In [28], the authors discussed the advantages of spark over the Hadoop map-reduce model. [29] 215 conducted a survey on the parallelization of density-based clustering algorithm for spatial data 216 mining based on spark. The authors of [30] conducted a performance evaluation of k-means over 
217 spark and map- reduce. On the other hand, a performance evaluation of three versions of k-means

218 clustering for biomedical data using spark was conducted in [31]. A performance evaluation of

219 parallel k-means with optimization algorithms for clustering big data using spark was conducted in

220 [32]. However, all the above surveys are either before 2016 or do not present a comprehensive

221 discussion on all types of clusters. Therefore, a comprehensive survey on clustering algorithms of

222 big data using Apache Spark is required to assess the current state-of-the-art and outline the future 223 directions of clustering big data.

224

225

226

227

228

229

230

231

232

233

234

235

236

237

238

239

240

\section{Survey Methodology}

The subject matter reviewed in this article is based on a literature review in clustering methods using Apache spark. We searched for the works regarding this topic and classify them into different Clustering techniques. All these papers talk about optimizing clustering techniques to solve the issues of big data clustering problems for various problems, viz., improve clustering accuracy, minimize execution time, increase throughput and scalability. Particularly, we are addressing the following questions:

- What are the types of Spark-based clustering methods?

- Which methods were used in the literature to cluster Big Data?

- What are the gaps in this research area?

- What optimization techniques were used in clustering?

- What are the pros and cons of the different Spark-based clustering methods?

\section{A. Search strategy}


241 To narrow the scope of the searching for relevant papers to be included in this study, we used the

242 "AND" and "OR" Boolean operators to combine the terms related to Spark-based clustering of Big

243 Data. The following terms are used to find the relevant papers.

244

- "Clustering big data using spark",

245

- "Apache Spark for Big data",

246

- "Clustering Big Data",

247

- "Clustering methods",

248

- “Data partitioning",

- “Big Data Partitioning",

250

- “Data segmentation".

251

252

The papers relevant to Spark-based clustering of Big Data were retrieved from the following 253 online sources.

254

- IEEE Explorer

255

- Springer,

256

- Elsevier

257

- ScienceDirect,

258

- Google Scholar,

259

- Researchgate,

260

261

262

\section{B. Paper filtering}


264 Initially 1230 and additional 43 reference books papers were identified through our search using 265 the previously explained research strategies. As shown in Fig. 2, 797 of these were eliminated via 266 our exclusion criteria. 476 papers were remaining. By reading and analysing the full-text articles, 267385 of them were excluded. Irrelevant papers were removed by applying the exclusion criteria 268 (shown below). In addition, duplicate papers retrieved from multiple sources were removed. 269 Finally, 91 articles were included in this survey. The following inclusion/exclusion rules are 270 applied on these papers.

271 - Inclusion criteria:

272 - papers published within the period from January 2010 to April 2020.

273 - papers in the area of Spark-based Big data clustering.

274 - papers written in English language.

275

276

277

278

- Exclusion criteria:

- papers on clustering but not on Big data.

- papers that are not using a Big data platform such as Spark. papers with no clear publication information, such as publisher, year, etc.

279

280

281

282

283

284

\section{Spark-based Clustering Algorithms}

In this work, the taxonomy of Spark-based Big Data clustering is developed to cover all the existing methods. Fig. 3 shows the developed taxonomy.

Survey Findings: The research questions (see Section IV) that we investigated in this survey 286 are addressed as shown below: 
287

288

289

290

291

292

293

294

295

296

297

298

299

300

301

302

303

304

305

306

307

308

- Answer to Q1: The Spark-based clustering algorithms were divided into three main categories: k-means based methods, hierarchal-based methods and density based methods. Each of these main categories were divided further into subcategories as depicted in Fig. 3. A detailed discussion of the Spark-based clustering methods in these subcategories is presented in the subsection below (Subsection V.A, V.B and V.C, Fig. 3 and Table 1).

- Answer to Q2: We discuss the different methods that have been proposed in the literature under each of the three main Spark-based clustering categories in Subsections V.A, V.B and V.C. The methods in these subsections are grouped based on their similarities in the approach. This grouping of the discussed methods is shown in Table 1.

- Answer to Q3: The gaps in the Spark-based clustering field are identified into two main points. The first is the lack of utilizing AI tools in clustering data and lack of using Big Data platforms. The second is that most current clustering methods do not support the characteristics of variety and velocity of Big Data. More discussion on this issue is in Section VI.

- Answer to Q4: Some existing works employed optimization techniques to improve clustering results. These optimization techniques were mainly used with k-means methods as discussed in subsection V.A.2 and V.D.

- Answer to Q5: The pros and cons of the different methods are discussed in the subsections V.A, V.B and VC that discuss the different types of Spark-based clustering methods. We also discuss our findings related to the Spark-based clustering methods in Section VI. 


\section{A. k-means based Clustering}

311 This method divides the data into disjoint clusters of similar points. In each cluster, a central

312 point is obtained via a distance function and is considered as the centroid of all other points within

313 the cluster. The clusters are iteratively optimized until an optimal solution is reached.

$314 \mathrm{k}$-mean is a framework of clustering or a family of distance functions, which provides the basis

315 for different variants of k-mean algorithms. $\mathrm{k}$ means is extensively used in clustering big data due

316 to its simplicity and fast convergence. One major backward of k-means is the priori setting of the

317 number of clusters, which have significant effect on the accuracy of final classification [33]. In

318 addition, k-means is not suited in situations where the clusters do not show convex distributed or 319 vary in sizes [34]. Due to these limitations, several modifications of $\mathrm{k}$-means have been proposed 320 such as fuzzy k-means and k-means++ [35]. Several works have been conducted to execute k321 means effectively under the Spark framework to improve its performance and scalability.

322 Therefore, the Spark-based k-means methods can be divided into four subcategories: Machine 323 Learning based methods, Fuzzy based methods, Statistics based methods and Scalable methods.

\section{A.1. Machine Learning based Methods}

325

326

327

328

329

330

331

332
The authors of [38] designed intelligent k-means based on spark. Intelligent k-means is a fully unsupervised learning that cluster data without any information regarding the number of clusters. A parallel implementation of biclustering using map-reduce over Spark platform was proposed by [41]. For improving the selection process of k-means, [47] combines Particle Swarm Optimization and Cuckoo-search to initiate better cluster centroid selections using spark framework. The work in [60] proposes a hybrid approach that integrate k-means and decision tree to cluster and detect anomaly in big data. At first, k-means is applied on the data to produce the clusters and then decision tree algorithm is applied on each cluster to classify normal and anomaly instances.

Peer] Comput. Sci. reviewing PDF | (CS-2020:08:51638:2:2:NEW 15 Oct 2020) 
anomalies using apache spark. Rule based is used for the detection of known attacked, while k-

means is used as unsupervised learning for the detection of new unknown attacks. Kdd cup dataset

was used to evaluate the algorithm and $93 \%$ accuracy was achieved. A paralleled algorithm for the

evolving clustering method was proposed by [63]. EMC is an online method which process one

data sample on a single pass and there is no iteration required to process the same data again.

These features make the algorithm highly efficient for processing contemporary real time applications where data arrive in a stream with high dimensionality. The authors evaluated the

proposed algorithm using massive credit card fraud dataset and the results show its superiority over

342 the traditional single EMC method.

343

344

345

346

347

348

349

350

351

352

353

354 355

\section{A.2. Fuzzy based Methods}

The authors of [45] proposed a parallel implementation of fuzzy consensus clustering for on the Spark platform for processing large scale heterogenous data. The authors of [46] developed a crime pattern-discovery system based on fuzzy clustering under Spark. The method uses L2 norm rather than Euclidian distance to optimize the distance computations. In another paper, the fuzzy clustering method is used under Spark to detect potential criminal patterns in large-scale spatiotemporal datasets [53]. In [54] the authors developed a parallel Fuzzy based image segmentation algorithm for handling big data in the agriculture field. At first, the images were converted to RGB and distributed to the available nodes in cloud. Then, the membership of pixel points to different cluster centroids were calculated. Finally, the centroids of the clusters are updated iteratively until an optimal solution is obtained. The performance of the algorithm was evaluated using the Spark platform and a significant reduction in execution time compared to Hadoop-based approach. The authors of [55] proposed an algorithm of fuzzy c-Means. The 
356 proposed algorithm is a modification of the Scalable Random Sampling with Iterative

357 Optimization (SRSIO-FCM). The highlighted characteristics of this research were the elimination

358 of the need for maintaining the membership matrix, which proved pivotal in reducing execution

359 time.

360

361

362

\section{A.3. Statistics based Methods}

363

The authors of [50] used Apache Spark to perform text clustering. Two algorithms were used: k-

364

means and LDA. LDA is Widely used technique for clustering high dimensional text data and it

produces considerably higher clustering accuracy than conventional k- means. In [57] the authors

used gaussian mixture model on spark MLlib to cluster the zika virus epidemic. The produced

clusters were useful to visualize the spread of the virus during the epidemic. The authors of [58]

implemented GMM clustering method under the framework of Spark. Gibbs sampling method is

used instead of Expectation Maximization algorithm to estimate the parameters of the model. The

efficiency of the algorithms was verified via multi-method comparison. The authors in [61]

presented a novel distributed gaussian based clustering algorithm for analysing the behaviour of

households in terms of energy consumption. Various factors such as weather conditions, type of day and time of the day were considered. The proposed algorithm under Spark shows a higher

374 accuracy than other standard regression methods for energy consumption forecasting.

\section{A.4. Scalable Methods}

376 A parallel implementation of $\mathrm{k}$ means algorithm over spark is proposed in [36]. The proposed

377 algorithm involves three strategies for seeding: 1) a subset of data is selected randomly for 378 partitioning. 2)sequentially selecting $\mathrm{k}$ instance based on probability. 3) stochastically selecting 
379 seeds in parallel. The efficiency of the proposed algorithm was demonstrated via experiments on 380 large scale text and UCI datasets. In another paper, the authors addressed the issue of pre381 determining the number of input clusters which is a present problem in most K-means methods by 382 automating the number of input clusters which resulted in better clustering quality when processing 383 large scale data [37].

384 In [39], the authors presented a scalable k-means algorithm based on spark streaming for 385 processing real time- data. The algorithm consists of two parts. One part runs an online algorithm over the stream data and obtains only statistically relevant information and another part that uses an offline algorithm on the results of the former to produce the actual clusters. Since the algorithm only retains statistically relevant information, it's possible to save more physical spaces. In addition, the proposed algorithm can explain the evolution of the data as all the needed information is retrievable from the stored statistical information.

391 The authors of [40] used k-means under Spark to cluster students' behaviors into different 392 categories using information gathered from universities' information system management. It is a powerful technique for performing simultaneous clustering of rows and Columns in a matrix data format. This method is used extensively for the study of genes expression. The authors of [42] clustered satellite images in an astronomy study using in k-means++ under the spark framework. In this paper, the authors simultaneously apply k-means multiple times with different initial 397 centroids and value of $\mathrm{k}$ under each iteration. The optimal value of $k$ is determined by clusters 398 validity index for all the executions. The work in [43] presented a Spark-based k-prototypes (SKP) 399 clustering method for mixed large-scale data analysis. The authors exploit the in-memory operations of Spark to reduce the consumption time of MRKP method. The method was evaluated 
401 using simulated and real datasets under Spark and Hadoop platform and the results show that

402 higher efficiency and scalability is achieved under Spark.

403

404 The authors of [44] implemented a Scalable Random Sampling for K-Prototypes Using Spark.

405 The algorithm randomly selects a small group of data points and approximate the cluster centers

406 from these data. As a result, the method perform computation for only small portion of the whole 407 data set, which result in a significant speedup of existing k-prototypes methods. A Parallel 408 Overlapping k-means algorithm (POKM) is proposed in [48]. This algorithm can perform parallel 409 clustering processes leading to non-disjoint partitioning of data. An implementation of parallel k410 means with triangle inequality based on spark is proposed in [49]. The method is an improved 411 version of k-means, which is supposed to speed up the process of analysis by skipping many point412 centre distance computations, which can be beneficial when clustering high dimensional data. The 413 authors of [51] implemented k-means with triangle inequality to reduce search time and avoid 414 redundant computation. The authors point out that the efficiency of k-means can be improved 415 significantly using triangle inequality optimisations. A distributed possibilistic c-means algorithm 416 is proposed in [52]. Possibilistic c means differ from other k-means techniques by assigning 417 probabilistic membership values in each cluster for every input point rather than assigning a point 418 to a single cluster.

419

The authors of [56] implemented an adaptive k-mean using Spark stream framework for real 421 time-anomaly detection in clouds virtual machines. The authors evaluated the method under Spark 422 and Storm in terms of the average delays of tuples during clustering and prediction and the results 423 indicate that Spark is significantly faster than Storm. [59] designed a framework for clustering and 
424 classification of big data. The framework integrates k-means and decision tree learning (ID3)

425 algorithms. the authors evaluated the framework under Spark in cluster of 37 nodes. the results 426 show that the proposed algorithms outperform spark machine learning library but is slightly slower 427 than the approximate k-means.

428

429

430

\section{B. Hierarchical Clustering}

431 This clustering technique is composed of two approaches: agglomerative and divisive. The first 432 approach considers every data point as a starter in its singleton cluster and the two nearest clusters are combined in each iteration until the two different points belong to a similar cluster. However, the second approach performs recursive top-down splitting. The existing hierarchical clustering methods can be divided into three subcategories: Data Mining based methods, Machine Learning based methods and Scalable methods.

\section{B.1. Data Mining based Methods}

438 A weighted agglomerative hierarchical clustering algorithm is introduced in [65]. The algorithm 439 was developed to analyse residents' activities in China. The data was based on the mobile phone's connection with the nearest stations, and within a week that data was collected and stored in Spark

441 for analysing. At first, hot areas where there are large population were identified, followed by an analysis of pedestrian's flow for each hot area. Meaningful information was obtained at less cost

443 and higher accuracy than the traditional method of investigation. The work in [66] proposed a 444 distributed-hierarchical based clustering algorithm that combines the features of the divisive and 445 agglomerative methods. The method consists of two operations. The first operation performs a 446 division on the domain of the dataset using the definition of binary space partition, which yields a 
447 set of coarse clusters that are then refined by identifying outliers and assigning remaining points to

448 nearest cluster. The second operation involves an agglomerative procedure over the previously

449 refined clusters. In [68], they proposed a Distributed-based Hierarchical Clustering System for

450 Large-Scale Semiconductor Wafers (DHCSSW) by applying the big data Spark framework to

451 existing hierarchical clustering algorithm.

\section{B.2. Machine Learning based Methods}

453 In [70], the authors designed clustering algorithms that can be used in MapReduce using Spark 454 platform. Particularly, they focus on the practical and popular serial Self-organizing Map clustering 455 algorithm (SOM). [71] proposed an algorithm called Spark-GHSOM, which scales to large realworld datasets on a distributed cluster. Moreover, it also proposed a new distance hierarchy approach for mixed attribute datasets and generated a multi-level hierarchy of SOM layers.

\section{B.3. Scalable Methods}

In [64], a parallel algorithm of Single-linkage Hierarchical Clustering was proposed by 462 formulating the problem as a Minimum Spanning Tree problem. The algorithm was evaluated using two large datasets with different distributions. The authors observed that spark is totally successful for the parallelization of linkage hierarchical clustering with acceptable scalability and high performance. The work in [67] proposed a system to detect anomaly for multi-source VMware-based cloud data center. The framework monitors VMware performance stream data 467 (e.g., CPU load, memory usage, etc.) continuously. The authors of [69] presented an incremental 468 hierarchical density-based stream clustering algorithm based on cluster stability. 


\section{Density-based Clustering}

473 Density-based clustering approaches in comparison with other types of clustering algorithms 474 have some superiorities, such as clustering arbitrary shape groups of data regardless of the 475 geometry and distribution of data, robustness to outliers, independence from the initial start point 476 of the algorithm, and its deterministic and consistent results in the repeat of the similar algorithm. 477 Motivated by these features, several studies have been conducted on the parallelization of Density 478 clustering method over Spark. Density-based clustering methods can be divided into four 479 subcategories: Graph based methods, Data Mining based methods, Machine Learning based 480 methods and Scalable methods.

481

482 483 484 485 486 487 488 489 490

\section{C.1. Graph based Methods}

In [74], the authors proposed a parallel implementation of density peaks clustering algorithm based on Spark's GraphX. The method was evaluated using spark and the results indicate that spark can perform up to 10x time faster compared to Hadoop map-reduce implementation. [79] proposed a distributed parallel algorithm of structure similarity clustering based on Spark (SparkSCAN) to cluster directed graph. Similarly, the authors of [80] exploited the advantage of the in-memory computation feature of spark to design a distributed network algorithm called CASS for clustering large-scale network based on structure similarity. Optimization approaches such as Bloom filter and shuffle selection are used to reduce memory usage and execution time. [82] designed a distributed algorithm that produces an approximate solution to the exact DBSCAN clustering. The method uses vertex-centric instead of Euclidean distance whereby a neighbourhood graph is computed. 


\section{C.2. Data Mining based Methods}

494 In another paper [76], the authors presented a fast parallel DBSCAN algorithm using Spark to 495 get around the shuffle operation. Each executor computes the partial clusters locally. The merging 496 process is deferred until all the partial clusters have been sent back to the driver. In [78] the authors 497 propose a scalable distributed density based hesitant fuzzy clustering for finding similar expression 498 between distinct genes. The proposed method benefits from the robustness of density-based 499 clustering against outliers and from the weighted correlation operators of hesitant fuzzy clustering 500 to measure similarity. The output clusters are based on the content of the neighbour graph. [83] 501 designed and implemented a scalable Shared Nearest Neighbours clustering called SparkSNN over 502 spark framework. Shared Nearest Neighbours is proven efficient for handling high-dimensional 503 spatiotemporal data. The algorithm was evaluated in terms of scalability and speed-up using 504 Marylanf crime data, the results demonstrated the effectiveness of the proposed algorithm.

\section{C.3. Machine Learning based Methods}

506

507 An algorithm based on adaptive density estimation is proposed for distributed big data approach 508 and tested on some prevalent datasets. This algorithm has no dependency, and every step of the 509 algorithm executes independently. Bayesian Locality Sensitive Hashing (LSH) is used to divide the 510 input data into partitions. The outliers are filtered out by locality preservation, which makes this 511 approach robust. The clusters are made very much homogenous via density definition on Ordered

512 Weighted Averaging distance [72]. A scalable distributed density-based clustering for performing 513 multi- regression tasks is proposed in [77]. In this work, locality sensitive hashing is used to enable 514 the algorithm to handle high dimensional data. A distributed clustering algorithm named 515 REMOLD is introduced in [84]. A two-step strategy has been applied in the REMOLD algorithm. 
516 In the first step, it uses the LSH partitioning method for balancing the effect of runtime and local

517 clustering while in the second step the partitions are clustered locally and independently using

518 Kernel-density and Higher-density nearest neighbour. Gaussian distribution is used to model the

519 local clusters. These models are eventually assembled at a central server to form the global 520 clusters.

\section{C.4. Scalable Methods}

522 In [73], a parallel implementation of DBSCAN algorithm ( $\mathrm{S}_{-}$DBSCAN) based on spark is

523 proposed. The algorithm is divided into three stages; partitioning the input data based on random

524 sampling; perform local DBSCAN in parallel to generate partial clusters; merge the partial clusters

525 based on the centroid. The algorithm can quickly realize the mergers and divisions of clustering

526 results from the original data. The authors compared the performance of their parallel algorithm

527 with a serial version on the Spark platform for massive data processing and an improvement in

528 performance was demonstrated. [75] proposed a scalable parallel implementation of DBSCAN

529 algorithm in Apache spark by applying a partitioning strategy. The algorithm uses a kd-tree in

530 order to reduce the search time. To achieve better performance and scalability, a partitioning

531 technique is applied to produce balanced sub-domains, which can be computed within Spark

532 executors. An implementation of DBSCAN algorithm using spark is proposed in [81]. Initially, a

533 pre-processing step is applied on the dataset to produce a set of representative points while

534 retaining the original data distribution and density information. This enables the algorithm to scale

535 up to large scale data. The new set is then used as an input to the algorithm for clustering. A real-

536 time density-based clustering algorithm (RT-DBSCAN) is proposed in [85]. RT-DBSCAN is an

537 extension of dbscan for supporting streamed data analysis. The algorithm employs the concept of 
538 spatiotemporal distance for clustering spatio-temporal data. The algorithm was implemented over

539 spark stream and evaluated using social media content.

540

541 D. Clustering Optimization

542 Some Spark-based clustering techniques, especially the k-means based methods, were supported

543 by optimization techniques to improve their clustering results. Due to the rise of AI based

544 computing in recent years, some research works have utilized AI tool in enhancing the clustering

545 methods while leveraging the benefits of Big Data platforms such as Spark. Other studies adapt

546 optimization techniques to improve the performance of clustering methods. [86] proposed a hybrid

547 method composed of PSO and k-means using apache spark. The diversity of the swarm ensures

548 that a global search is conducted, hence, the resulting cluster centroids are not dependent on the

549 initial choice. The approach was compared with stand-alone k-means and it showed better

550 performance in terms of convergence. [87] proposed an adaptive swarm-based clustering for

551 stream processing of twitter data. Initially, fuzzy c-means is applied as pre-processing step to

552 produce the initial cluster centres, then the clusters are further optimized using adaptive particle

553 swarm optimization.

554 The authors of [88] and [89] combined the robust artificial bee colony algorithm with the

555 powerful Spark framework for large scale data analysis. The characteristics of $\mathrm{ABC}$ makes the 556 algorithms avoid local minimum while Spark in memory computation accelerates the speed of

557 computation and convergence time. The KDD CUP 99 data was utilized to verify the effectiveness

558 of the method. The experimental results show that the algorithm produce high clustering quality

559 and nearly as fast as the serial algorithms. Other unsupervised learning such as self-organised map 
560 has also been proposed [90]. To tackle high dimensional data, subspace clustering was proposed by $561 \quad[91]$.

\section{VI. Discussion and Future Direction}

563 From the discussion of the previous section, we note that most existing methods (see Table 1) have

564 addressed the volume characteristic of the Big Data used in their experiments. However, few

565 existing methods have shown that their methods support the variety and velocity characteristics of

566 the used Big Data. Additionally, most methods used real Big Data validate their proposed

567 methods as seen in Table 1. From Table 1, we conclude that there is a lot of room for research in

568 clustering methods to support the characteristics of variety and velocity of Big data since only few

569 works have addressed these issues.

570 A fundamental assumption of most clustering algorithms is that all data features are considered

571 equally important. However, such approach often fails in high dimensional space. A subspace

572 clustering overcome the issue of high dimensional data by establishing a set of features that it

573 supposes to be most significant for each cluster.

574 Since the Big data platforms were only developed in the last few years, the existing clustering 575 problems adapted to such platforms were extensions of the traditional clustering techniques.

576 Researchers are yet to develop clustering techniques that are native to the Big Data platforms such

577 as Spark. The research direction of adapting the optimization techniques such as PSA, Bee colony 578 and $\mathrm{ABC}$ to smoothly work with Spark is yet to be investigated by researchers who are interested 579 in clustering Big Data. Another area of research that is has not been fully investigated is adopting 580 Fuzzy-based clustering algorithms on Spark.

581 In general, due to the infancy of Spark-based clustering algorithms, only few researchers 582 attempted designing techniques that leverage the potential of parallelism of Spark in cluster Big 
583 Data. In the coming years, we foresee a large influx of research works in this important area of

584 Spark-based clustering of Big Data. Particularly, there are ample opportunities in future research to 585 utilize AI tools in clustering data while leveraging the benefits of Big Data platforms such as 586 Spark.

587 In Table 2, we note that most of the papers used in this survey were extracted from the IEEE 588 Explorer. However, the other data sources shown in Table 2 were of great benefit to this survey. 589 An interesting finding was shown in Table 3, where most the existing Spark-based Clustering were 590 published in the years 2016-2019. This indicates that clustering methods that leverage Big Data 591 platforms is still in its early days and there is a lot of potential of research in this area.

592 In summary, we highlight three new research directions:

593 - Utilizing AI tools in clustering data while leveraging the benefits of Big Data platforms $594 \quad$ such as Spark.

595 - Clustering methods to support the characteristics of variety and velocity of Big data.

596 Additionally, support new aspects of clustering such as concept drift, scalability, 597 integration, fault-tolerance, consistency, timeliness, load balancing, privacy, and 598 incompleteness, etc.

599 - Clustering methods to utilize Spark as it is an efficient Big Data platform.

600

601

602

603 VII. Conclusions

604

605 As a consequence of the spread of smart devices and appearance of new technologies such as 606 IoT, huge data have been produced on daily bases. As a result, the concept of Big Data has 
607 appeared. Unlike the traditional clustering approaches, Big Data clustering requires advanced

608 parallel computing for better handling of data because of the enormous volume and complexity.

609 Therefore, this work contributes to the research in this area by providing a comprehensive

610 overview of existing Spark-based clustering techniques on Big data and outlines some future

611 directions in this area.

612 Due to the infancy of the Big data platforms such as Spark, the existing clustering techniques

613 that are based on Spark are only extensions of the traditional clustering techniques. There is still

614 big room for developing clustering techniques designed specifically for Spark making use of the

615 random distribution of data onto Spark partitions, called RDDs, and the parallel computation of

616 data in the individual RDDs. Through this survey we found that most existing Spark-based

617 clustering method support the volume characteristic of Big Data ignoring other characteristics.

618 Therefore, future research should focus on other characteristics as well such as variety and

619 velocity. Additionally, future Spark-based clustering method should investigate new features such

620 as concept drift, scalability, integration, fault-tolerance, consistency, timeliness, load balancing,

621 privacy, etc.

622

623

624 Acknowledgements

625 The authors would like to thank the Deanship of Scientific Research at Prince Sattam Bin 626 Abdulaziz University for their support of this research

627

628

629

630

631

632

633

634

635

\section{References}

[1] Bhadani, A. K., \& Jothimani, D. (2016). Big Data: Challenges, Opportunities, and Realities. In Singh, M. K., \& G., D. K. (Ed.), Effective Big Data Management and Opportunities for Implementation (pp. 1-24).

[2] Dave, M. and Gianey, H. "Different clustering algorithms for Big Data analytics: A review," 2016 International Conference System Modeling \& Advancement in Research Trends (SMART), Moradabad, 2016, pp. 328-333, doi: 10.1109/SYSMART.2016.7894544. 
636

637

638

639

640

641

642

643

644

645

646

647

648

649

650

651

652

653

654

655

656

657

658

659

660

661

662

663

664

665

666

667

668

669

670

671

672

673

674

675

676

677

678

679

680

681

682
[3] Othman, S.M., Ba-Alwi, F.M., Alsohybe, N.T. Al-Hashida, A.Y.. Intrusion detection model using machine learning algorithm on Big Data environment. J Big Data 5, 34 (2018). https://doi.org/10.1186/s40537-018$\underline{0145-4}$.

[4] Hu, S., Xiao, Z., Rao, Q., and Liao, R. "An anomaly detection model of user behavior based on similarity clustering," 2018 IEEE 4th Information Technology and Mechatronics Engineering Conference (ITOEC), Chongqing, China, 2018, pp. 835-838, doi: 10.1109/ITOEC.2018.8740748.

[5] Fasheng, L. and Xiong, L. "Survey on text clustering algorithm -Research present situation of text clustering algorithm," 2011 IEEE 2nd International Conference on Software Engineering and Service Science, Beijing, 2011, pp. 196-199, doi: 10.1109/ICSESS.2011.5982288.

[6] Baltas A., Kanavos A., Tsakalidis A.K. An Apache Spark Implementation for Sentiment Analysis on Twitter Data. In: Sellis T., Oikonomou K. (eds) Algorithmic Aspects of Cloud Computing. ALGOCLOUD 2016. Lecture Notes in Computer Science, vol 10230. Springer, Cham.

[7] Ajin. V. W. and Kumar, L. D., "Big data and clustering algorithms," 2016 International Conference on Research Advances in Integrated Navigation Systems (RAINS), Bangalore, 2016, pp. 1-5, doi: 10.1109/RAINS.2016.7764405.

[8] Shoro, S. A. G. and Soomro, T. R., "Big data analysis: Apache Spark perspective," Global Journal of Computer Science and Technology, vol. 15, no. 1, 2015.

[9] Verma, A., Mansuri, A. H., and Jain, N., "Big data management processing with Hadoop MapReduce and spark technology: A comparison," 2016 Symposium on Colossal Data Analysis and Networking (CDAN), Indore, 2016, pp. 1-4, doi: 10.1109/CDAN.2016.7570891.

[10] Labrinidis, A. and Jagadish, H. V.,.. Challenges and opportunities with big data. Proc. VLDB Endow. 5, 12 (August 2012), 2032-2033. DOI:https://doi.org/10.14778/2367502.2367572.

[11] Gousios, G,. 2018. Big data software analytics with Apache Spark. In Proceedings of the 40th International Conference on Software Engineering: Companion Proceeedings(ICSE '18). Association for Computing Machinery, New York, NY, USA, 542-543. DOI:https://doi.org/10.1145/3183440.3183458.

[12] Aziz K., Zaidouni D., Bellafkih M. Big Data Optimisation Among RDDs Persistence in Apache Spark. In: Tabii Y., Lazaar M., Al Achhab M., Enneya N. (eds) Big Data, Cloud and Applications. BDCA 2018. Communications in Computer and Information Science, vol 872. Springer, Cham.

[13] Salloum, S., Dautov, R., Chen, X., Peng, P. X., and Huang, J. Z.,. Big data analytics on apache spark. International Journal of Data Science and Analytics, 1(3):145-164, 2016.

[14] Mishra, D. D., Pathan, S. and Murthy, C., "Apache Spark Based Analytics of Squid Proxy Logs," 2018 IEEE International Conference on Advanced Networks and Telecommunications Systems (ANTS), Indore, India, 2018, pp. 1-6, doi: 10.1109/ANTS.2018.8710044.

[15] Kim, K.. Real-time Streaming Data Analysis using Spark. International Journal of Emerging Trends in Engineering Research. Volume 6, 2018.

[16] Assefi, M., Behravesh, E., Liu G. and Tafti, A. P., "Big data machine learning using apache spark MLlib," 2017 IEEE International Conference on Big Data (Big Data), Boston, MA, 2017, pp. 3492-3498, doi: 10.1109/BigData.2017.8258338.

[17] Armbrust, M. M., Xin, R. S., Lian, C., Huai, Y., Liu, D., Bradley, J. K., Meng, X., Kaftan, T., Franklin, M. J., Ghodsi, A. and Zaharia, M.,. Spark SQL: Relational Data Processing in Spark. In Proceedings of the 2015 ACM SIGMOD International Conference on Management of Data(SIGMOD '15). Association for Computing Machinery, New York, NY, USA, 1383-1394.

[18] Xin, R. S., Gonzalez, J. E., Franklin, M. J., and Stoica, I., “Graphx: Aresilient distributed graph system on spark," in First InternationalWorkshop on Graph Data Management Experiences and Systems,2013, pp. $2: 1-2: 6$.

[19] Xu, D. and Tian, Y., “A Comprehensive Survey of Clustering Algorithms,"Annals of Data Science, vol. 2, no. 2, pp. 165-193, 2015. 
683

684

685

686

687

688

689

690

691

692

693

694

695

696

697

698

699

700

701

702

703

704

705

706

707

708

709

710

711

712

713

714

715

716

717

718

719

720

721

722

723

724

725

726

727

728

729

730

731
[20] Zerhari, B.; Lahcen, A.A.; Mouline, S. Big data clustering: Algorithms and challenges. In Proceedings of the International Conference on Big Data, Cloud and Applications, Tetuan, Morocco, 25-26 May 2015

[21] Shirkhorshidi A.S., Aghabozorgi S., Wah T.Y., Herawan T. Big Data Clustering: A Review. In: Murgante B. et al. (eds) Computational Science and Its Applications - ICCSA 2014. ICCSA 2014. Lecture Notes in Computer Science, vol 8583. Springer, Cham

[22] Rotsnarani Sethy, Mrutyunjaya Panda, "Big Data Analysis using Hadoop: A Survey" in Proc. Conf. International Journal of Advanced Research in Computer Science and Software Engineering, Volume 5, Issue 7, July 2015.

[23] Bhandari, Rujal \& Dabhi, Dipak. (2016). Extensive Survey on k-Means Clustering using MapReduce in Datamining. Conference: International Conference on Electronics and Communication Systems (ICECS) At: Coimbatore, Tamilnadu, India.

[24] S Sood, Akshay \& Singh, Ravinder. (2019). A Survey of Performance Improvement Techniques for Hadoop. International Journal of Applied Engineering Research, ISSN 0973-4562 Vol. 10 No.55 (2015).

[25] M. Manwal and A. Gupta, "Big data and Hadoop - A technological survey," 2017 International Conference on Emerging Trends in Computing and Communication Technologies (ICETCCT), Dehradun, 2017, pp. 1-6.

[26] D. Jiang, B. Ooi, L. Shi, and S. Wu, "Big Data Processing Using Hadoop: Survey on Scheduling," Proc. VLDB Endow. vol. 3, no. 10, pp. 272-277, 2010.

[27] Tang, Shanjiang \& He, Bingsheng \& Yu, Ce \& Li, Yusen \& Li, Kun. (2018). A Survey on Spark Ecosystem for Big Data Processing.

[28] R. C. Maheshwar and D. Haritha, "Survey on high performance analytics of big data with apache spark," 2016 International Conference on Advanced Communication Control and Computing Technologies (ICACCCT), Ramanathapuram, 2016, pp. 721-725, doi: 10.1109/ICACCCT.2016.7831734.

[29] F. Huang, Q. Zhu, J. Zhou, J. Tao, X. Zhou, D. Jin, X. Tan, and L. Wang, "Research on the Parallelization of the DBSCAN Clustering Algorithm for Spatial Data Mining Based on the Spark Platform," Remote Sensing, vol. 9, no. 12, p. 1301, Dec. 2017.

[30] S. Ketu and S. Agarwal, "Performance enhancement of distributed k-Means clustering for big Data analytics through in-memory computation," 2015 Eighth International Conference on Contemporary Computing (IC3), Noida, 2015, pp. 318-324, doi: 10.1109/IC3.2015.7346700.

[31] A. Shobanadevi and G. Maragatham, "Studying the performance of clustering techniques for biomedical data using spark," 2017 International Conference on Intelligent Sustainable Systems (ICISS), Palladam, 2017, pp. 58-65, doi: 10.1109/ISS1.2017.8389249.

[32] Santhi V., Jose R. (2018) Performance Analysis of Parallel k-Means with Optimization Algorithms for Clustering on Spark. In: Negi A., Bhatnagar R., Parida L. (eds) Distributed Computing and Internet Technology. ICDCIT 2018. Lecture Notes in Computer Science, vol 10722. Springer, Cham.

[33] Hartigan J.A, Wong M.A., Algorithm A.S., A k-means clustering algorithm. Journal of the Royal Statistical Society Series C. 1979;28(1):100-108.

[34] Jain A.K. Data clustering: 50 years beyond k-means. Pattern Recognition Letters. 2010;31(8):651-666

[35] Huang Z., Extensions to the k-means algorithm for clustering large data sets with categorical values. Data mining and knowledge discovery. 1998;2(3):283-304

[36] Wang, B., Yin, J., Hua, Q., Wu Z. and Cao, J., "Parallelizing k-Means-Based Clustering on Spark," 2016 International Conference on Advanced Cloud and Big Data (CBD), Chengdu, 2016, pp. 31-36, doi: 10.1109/CBD.2016.016.

[37] Sinha A., and Jana, P. K., "A novel k-means based clustering algorithm for big data," International Conference on Advances in Computing, Communications and Informatics (ICACCI), Jaipur, 2016, pp. 1875-1879.

[38] Kusuma, I., Ma'sum, M. A., Habibie, N., Jatmiko W. and Suhartanto, H., "Design of intelligent k-means based on spark for big data clustering," International Workshop on Big Data and Information Security (IWBIS), Jakarta, 2016, pp. 89-96, doi: 10.1109/IWBIS.2016.7872895. 
[39] Backhoff, O. and Ntoutsi, E., "Scalable Online-Offline Stream Clustering in Apache Spark," IEEE 16th International Conference on Data Mining Workshops (ICDMW), Barcelona, 2016, pp. 37-44, doi: 10.1109/ICDMW.2016.0014.Parallel Implementation of Density Peaks Clustering Algorithm Based on Spark

[40] Ding, D., Li, J., Wang H. and Liang, Z., "Student Behavior Clustering Method Based on Campus Big Data," 13th International Conference on Computational Intelligence and Security (CIS), Hong Kong, 2017, pp. 500-503, doi: 10.1109/CIS.2017.00116.

[41] Sarazin, T., Lebbah M. and Azzag, H., "Biclustering using Spark-MapReduce," IEEE International Conference on Big Data (Big Data), Washington, DC, 2014, pp. 58-60, doi: 10.1109/BigData.2014.7004493.

[42] Sharma, T., Shokeen, V., Mathur, D.,, Multiple k-Means++ Clustering of Satellite Image Using Hadoop MapReduce and Spark. Published in International Journal of Advanced Studies in Computer Science and Engineering, IJASCSE volume 5 issue 4, 2016

[43] Ben HajKacem, M. A., Ben N'Cir, C. E. and Essoussi, N., "KP-S: A Spark-Based Design of the KPrototypes Clustering for Big Data," IEEE/ACS 14th International Conference on Computer Systems and Applications (AICCSA), Hammamet, 2017, pp. 557-563, doi: 10.1109/AICCSA.2017.94.

[44] Ben HajKacem M.A., Ben N'cir CE., Essoussi N. Scalable Random Sampling K-Prototypes Using Spark. In: Ordonez C., Bellatreche L. (eds) Big Data Analytics and Knowledge Discovery. DaWaK 2018. Lecture Notes in Computer Science, vol 11031. Springer, Cham.

[45] Wu, J., Wu, Z., Cao, J., Liu, H., Chen G. and Zhang, Y., "Fuzzy Consensus Clustering With Applications on Big Data," in IEEE Transactions on Fuzzy Systems, vol. 25, no. 6, pp. 1430-1445, Dec. 2017, doi: 10.1109/TFUZZ.2017.2742463.

[46] Win, K.N., Chen, J., Chen, Y. Fournier-Viger, P.. PCPD: A Parallel Crime Pattern Discovery System for Large-Scale Spatiotemporal Data Based on Fuzzy Clustering. Int. J. Fuzzy Syst. 21, 1961-1974 (2019). https://doi.org/10.1007/s40815-019-00673-3.

[47] Gao, Z. Q. and Zhang, L. J., "DPHKMS: An efficient hybrid clustering preserving differential privacy in spark," in International Conference on Emerging Internetworking, Data \& Web Technologies, 2017, pp.

[48] Zayani, A., Ben N'Cir, C. and Essoussi, N., "Parallel clustering method for non-disjoint partitioning of large-scale data based on spark framework," IEEE International Conference on Big Data (Big Data), Washington, DC, 2016, pp. 1064-1069.

[49] Chitrakar A. S., and Petrovic, S., "Analyzing Digital Evidence Using Parallel k-means with Triangle Inequality on Spark," IEEE International Conference on Big Data (Big Data), Seattle, WA, USA, 2018, pp. 3049-3058, doi: 10.1109/BigData.2018.8622430.

[50] Shah, J.,"New Clustering Using Spark", International Journal of Latest Technology in Engineering, Management \& Applied Science (IJLTEMAS) Volume V, Issue IX, 2016.

[51] Fatta, G. and Al Ghamdi, S., Efficient Clustering Techniques on Hadoop and Spark. International Journal of Big Data Intelligence. Vol. 6, 3-4, 269-290, 2019.

[52] Zhang, Y., Liu, H., Chen, T. and Tang, Di., A Distributed PCM Clustering Algorithm Based on Spark. In Proceedings of the 2019 11th International Conference on Machine Learning and Computing (ICMLC '19). Association for Computing Machinery, New York, NY, USA, 70-74. DOI:https://doi.org/10.1145/3318299.3318315

[53] Win, K. N., Chen, J., Xiao, G., Chen Y. and Viger, P. F., "A Parallel Crime Activity Clustering Algorithm Based on Apache Spark Cloud Computing Platform," IEEE 21st International Conference on High Performance Computing and Communications; Zhangjiajie, China, 2019, pp. 68-74, doi: 10.1109/HPCC/SmartCity/DSS.2019.00025.

[54] Liu, B., He, S., He, D., Zhang Y. and Guizani, M., "A Spark-Based Parallel Fuzzy c -Means Segmentation Algorithm for Agricultural Image Big Data," in IEEE Access, vol. 7, pp. 42169-42180, 2019, doi: 10.1109/ACCESS.2019.2907573. 
780

781

782

783

784

785

786

787

788

789

790

791

792

793

794

795

796

797

798

799

800

801

802

803

804

805

806

807

808

809

810

811

812

813

814

815

816

817

818

819

820

821

822

823

824

825

826

827
[55] Bharill, N., Tiwari, A.,and Malviya, A., Fuzzy Based Scalable Clustering Algorithms for Handling Big Data Using Apache Spark. IEEE Transactions on Big Data. 2016, PP. 1-1. 10.1109/TBDATA.2016.2622288.

[56] Solaimani, M., Iftekhar, M., Khan, L., Thuraisingham B. and Ingram, J. B., "Spark-based anomaly detection over multi-source VMware performance data in real-time," IEEE Symposium on Computational Intelligence in Cyber Security (CICS), Orlando, FL, 2014, pp. 1-8, doi: 10.1109/CICYBS.2014.7013369.

[57] Lavanya, K., Sairabanu, J., \& Jain, P. Clustering of Zika virus epidemic using Gaussian mixture model in spark environment. Biomedical Research-tokyo, vol 30, pp. 127-133, 2019.

[58] Pang H., Deng L., Wang L., Fei M. The Application of Spark-Based Gaussian Mixture Model for Farm Environmental Data Analysis. In: Zhang L., Song X., Wu Y. (eds) Theory, Methodology, Tools and Applications for Modeling and Simulation of Complex Systems. AsiaSim 2016, SCS AutumnSim 2016. Communications in Computer and Information Science, vol 645. Springer, Singapore

[59] Mallios X., Vassalos V., Venetis T., Vlachou A. A Framework for Clustering and Classification of Big Data Using Spark. In: Debruyne C. et al. (eds) On the Move to Meaningful Internet Systems: OTM 2016 Conferences. OTM 2016. Lecture Notes in Computer Science, vol 10033. Springer, Cham

[60] Thakur S., Dharavath R. KMDT: A Hybrid Cluster Approach for Anomaly Detection Using Big Data. In: Satapathy S., Tavares J., Bhateja V., Mohanty J. (eds) Information and Decision Sciences. Advances in Intelligent Systems and Computing, vol 701. Springer, Singapore, 2018.

[61] Chakravorty, A., Rong, C., Evensen P. and Wlodarczyk, T. W., "A distributed gaussian-means clustering algorithm for forecasting domestic energy usage," International Conference on Smart Computing, Hong Kong, 2014, pp. 229-236, doi: 10.1109/SMARTCOMP.2014.7043863.

[62] Lighari, S. N. and Hussain, D. M. A., "Hybrid model of rule based and clustering analysis for big data security," 2017 First International Conference on Latest trends in Electrical Engineering and Computing Technologies (INTELLECT), Karachi, 2017, pp. 1-5, doi: 10.1109/INTELLECT.2017.8277627.

[63] Kamaruddin S., Ravi V., Mayank P. Parallel Evolving Clustering Method for Big Data Analytics Using Apache Spark: Applications to Banking and Physics. In: Reddy P., Sureka A., Chakravarthy S., Bhalla S. (eds) Big Data Analytics. BDA 2017. Lecture Notes in Computer Science, vol 10721. Springer, Cham.

[64] Jin, C., Liu, R., Chen, Z., Hendrix, W., Agrawal A. and Choudhary, A., "A Scalable Hierarchical Clustering Algorithm Using Spark," 2015 IEEE First International Conference on Big Data Computing Service and Applications, Redwood City, CA, 2015, pp. 418-426, doi: 10.1109/BigDataService.2015.67.

[65] Guo, Y., Zhang J. and Zhang, Y., "An Algorithm for Analyzing the City Residents' Activity Information through Mobile Big Data Mining," IEEE Trustcom/BigDataSE/ISPA, Tianjin, 2016, pp. 2133-2138, doi: 10.1109/TrustCom.2016.0328.

[66] Ianni, M., Masciari, E., Mazzeo, G.M., Mezzanzanica, M., Zaniolo, C., Fast and effective Big Data exploration by clustering. Future Generation Computer Systems Volume 102, Pages 84-94, 2020.

[67] Solaimani, M., Iftekhar, M., Khan, L., Thuraisingham, B., and Ingram, J. B., Spark-based anomaly detection over multi-source VMware performance data in real-time. In 2014 IEEE Symposium on Computational Intelligence in Cyber Security (CICS) (pp. 1-8).

[68] Lee, S., and Kim, D. Distributed-based hierarchical clustering system for large-scale semiconductor wafers. In 2018 IEEE International Conference on Industrial Engineering and Engineering Management (IEEM) (pp. 1528-1532).

[69] Hassani, M., Spaus, P., Cuzzocrea, A., and Seidl, T., I-hastream: density-based hierarchical clustering of big data streams and its application to big graph analytics tools. In 2016 16th IEEE/ACM International Symposium on Cluster, Cloud and Grid Computing (CCGrid) (pp. 656-665).

[70] Sarazin, T., Azzag, H., and Lebbah, M., SOM clustering using spark-mapreduce. In 2014 IEEE International Parallel \& Distributed Processing Symposium Workshops (pp. 1727-1734).

[71] Malondkar, A., Corizzo, R., Kiringa, I., Ceci, M., \& Japkowicz, N., Spark-GHSOM: Growing Hierarchical Self-Organizing Map for large scale mixed attribute datasets. Information Sciences, 496, 572-591, 2019. 
828

829

830

831

832

833

834

835

836

837

838

839

840

841

842

843

844

845

846

847

848

849

850

851

852

853

854

855

856

857

858

859

860

861

862

863

864

865

866

867

868

869

870

871

872

873

874
[72] Hosseini, B. and Kiani, K.., A Robust Distributed Big Data Clustering-based on Adaptive Density Partitioning using Apache Spark. Symmetry., 2018. 10. 342. 10.3390/sym10080342.

[73] Luo, G., Luo, X., Gooch, T. F., Tian L. and Qin, K., "A Parallel DBSCAN Algorithm Based on Spark," IEEE International Conferences on Big Data and Cloud Computing (BDCloud), Social Computing and Networking (SocialCom), Sustainable Computing and Communications (SustainCom) (BDCloudSocialCom-SustainCom), Atlanta, GA, 2016, pp. 548-553, doi: 10.1109/BDCloud-SocialComSustainCom.2016.85.

[74] Rui, L., Xiaoge, L., Liping, D., Shuting, Z. and Mian, W., Parallel Implementation of Density Peaks Clustering Algorithm Based on Spark, procedia Computer Science, Volume 107, 2017, Pages 442-447.

[75] Han, D., Agrawal, A., Liao, W. and Choudhary, A., "Parallel DBSCAN Algorithm Using a Data Partitioning Strategy with Spark Implementation," 2018 IEEE International Conference on Big Data (Big Data), Seattle, WA, USA, 2018, pp. 305-312, doi: 10.1109/BigData.2018.8622258.

[76] Han D., Agrawal A., Liao W., Choudhary A. A Fast DBSCAN Algorithm with Spark Implementation. In: Roy S., Samui P., Deo R., Ntalampiras S. (eds) Big Data in Engineering Applications. Studies in Big Data, vol 44. Springer, Singapore, 2018.

[77] Corizzo, R., Pio, G., Ceci, M. Malerba, D.. DENCAST: distributed density-based clustering for multi-target regression. J Big Data 6, 43 (2019). https://doi.org/10.1186/s40537-019-0207-2

[78] Hosseini, B. and Kourosh K., "A big data driven distributed density based hesitant fuzzy clustering using Apache spark with application to gene expression microarray." Eng. Appl. Artif. Intell. 79 (2019): 100-113.

[79] Zhou Q., Wang J. SparkSCAN: A Structure Similarity Clustering Algorithm on Spark. In: Chen W. et al. (eds) Big Data Technology and Applications. BDTA 2015. Communications in Computer and Information Science, vol 590. Springer, Singapore

[80] Kim, J., Shin, M., Kim, J., Park, C., Lee, S., Woo, J., ... \& Park, S. "CASS: A distributed network clustering algorithm based on structure similarity for large-scale network." PloS one vol. 13,10 e0203670. 10 Oct. 2018, doi:10.1371/journal.pone.0203670

[81] Baralis, E., Garza P. and Pastor, E., "A Density-based Preprocessing Technique to Scale Out Clustering," 2018 IEEE International Conference on Big Data (Big Data), Seattle, WA, USA, 2018, pp. 2078-2087, doi: 10.1109/BigData.2018.8621870.

[82] Lulli, A., Dell'Amico, M. and Ricci, L., NG-DBSCAN: scalable density-based clustering for arbitrary data. Proceedings of the VLDB Endowment. Vol 10, pp. 157-168, 2016.

[83] Aryal, A. M. and Wang, S., "SparkSNN: A density-based clustering algorithm on spark," IEEE 3rd International Conference on Big Data Analysis (ICBDA), Shanghai, 2018, pp. 433-437, doi: 10.1109/ICBDA.2018.8367722.

[84] Liang, M., Li, Q., Geng, Y., Wang, J. and Wei, Z., "REMOLD: An Efficient Model-Based Clustering Algorithm for Large Datasets with Spark," 2017 IEEE 23rd International Conference on Parallel and Distributed Systems (ICPADS), Shenzhen, 2017, pp. 376-383.

[85] Gong, Y., Sinnott, R.O., Rimba, P. RT-DBSCAN: Real-Time Parallel Clustering of Spatio-Temporal Data Using Spark-Streaming. In: Shi Y. et al. (eds) Computational Science - ICCS 2018. ICCS 2018. Lecture Notes in Computer Science, vol 10860. Springer, Cham.

[86] Sherar, M., and Zulkernine, F., "Particle swarm optimization for large-scale clustering on apache spark," 2017 IEEE Symposium Series on Computational Intelligence (SSCI), Honolulu, HI, 2017, pp. 1-8, doi: 10.1109/SSCI.2017.8285208.

[87] Hasan, R.A., Alhayali, R.A., Zaki, N.D., \& Ali, A.H. An adaptive clustering and classification algorithm for Twitter data streaming in Apache Spark. TELKOMNIKA Telecommunication Computing Electronics and Control, vol 17, pp. 3086-3099, 2019.

[88] Wang, Y. and Q. Qian, "A Spark-Based Artificial Bee Colony Algorithm for Large-Scale Data Clustering," IEEE 20th International Conference on High Performance Computing and Communications; IEEE 16th 
875

876

877

878

879

880

881

882

883

884

885

886

887
International Conference on Smart City; IEEE 4th International Conference on Data Science and Systems (HPCC/SmartCity/DSS), Exeter, United Kingdom, 2018, pp. 1213-1218, doi: 10.1109/HPCC/SmartCity/DSS.2018.00204.

[89] Bonab, M.B., Hashim, S.Z.M., Alsaedi, A.K.Z., and Hashim, U.R. Modified k-means Combined with Artificial Bee Colony Algorithm and Differential Evolution for Color Image Segmentation. In: PhonAmnuaisuk S., Au T. (eds) Computational Intelligence in Information Systems. Advances in Intelligent Systems and Computing, vol 331, 2015, Springer, Cham

[90] Sarazin, T., Azzag, H. and Lebbah, M., "SOM Clustering Using Spark-MapReduce," 2014 IEEE International Parallel \& Distributed Processing Symposium Workshops, Phoenix, AZ, 2014, pp. 17271734, doi: 10.1109/IPDPSW.2014.192.

[91] Sembiring, R. W. and Jasni M. Z., \& Embong, A., Clustering High Dimensional Data Using Subspace and Projected Clustering Algorithms. International Journal of Computer Science \& Information Technology, 2010. 2. 10.5121/ijcsit.2010.2414. 
Figure 1

Apache Spark Architecture

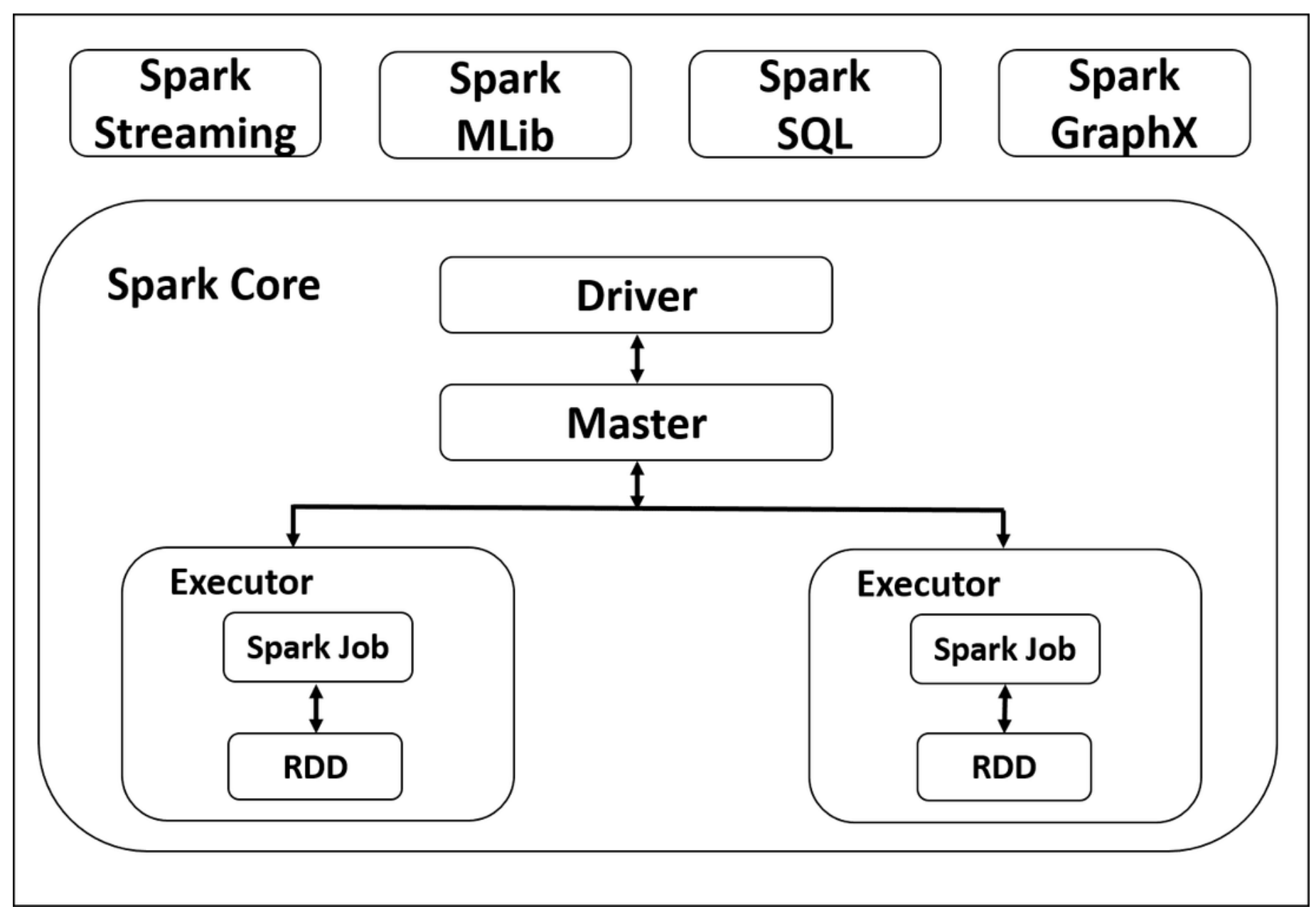


Figure 2

Flowchart for paper exclusion 

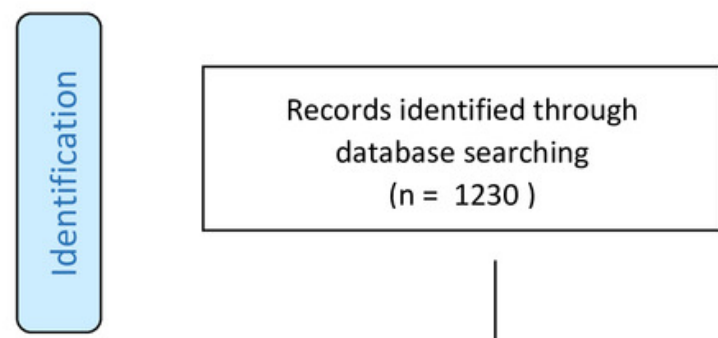
Additional records identified through other sources

$(n=1230)$

$(n=43)$
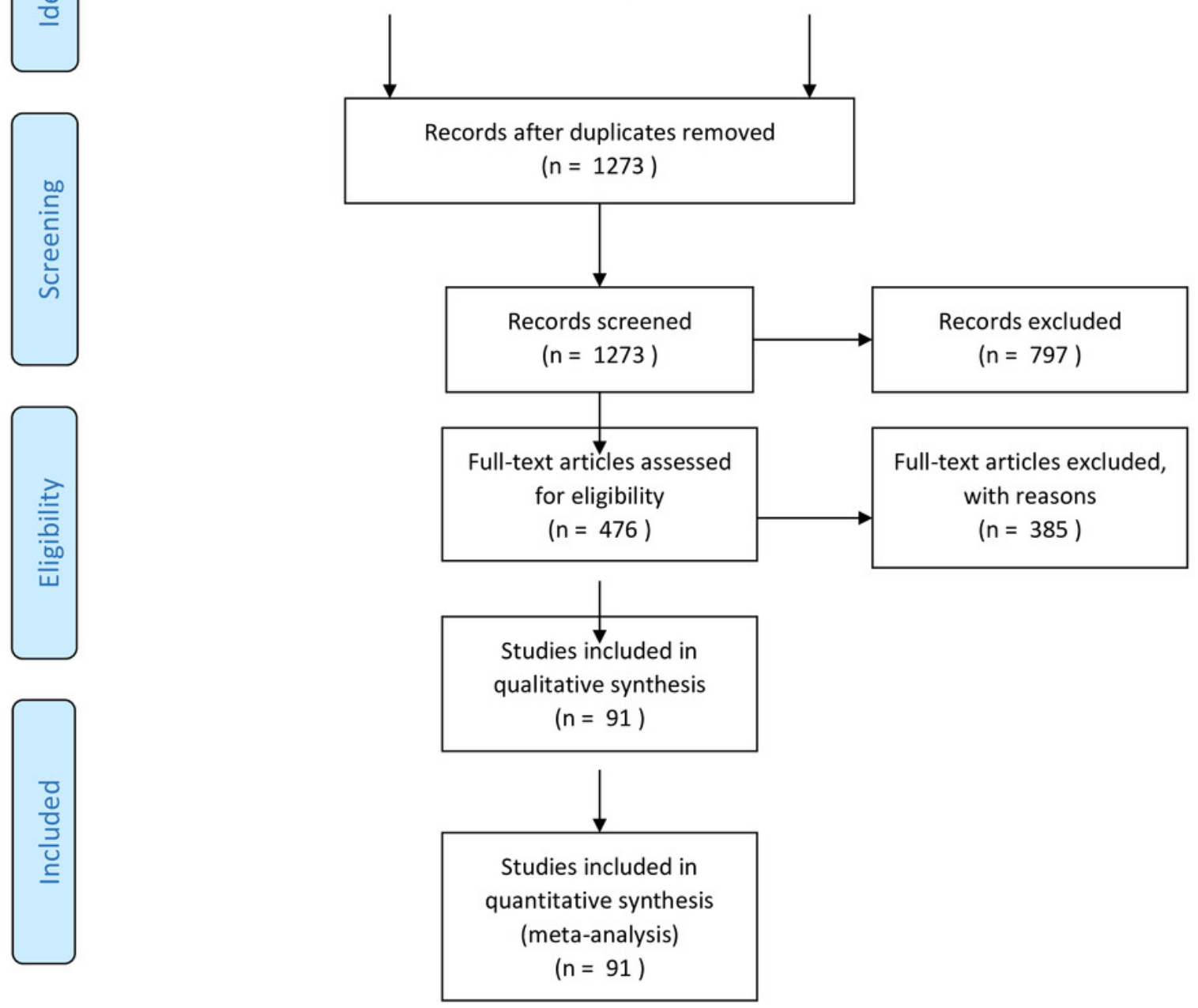
Figure 3

Taxonomy of Spark-based clustering methods

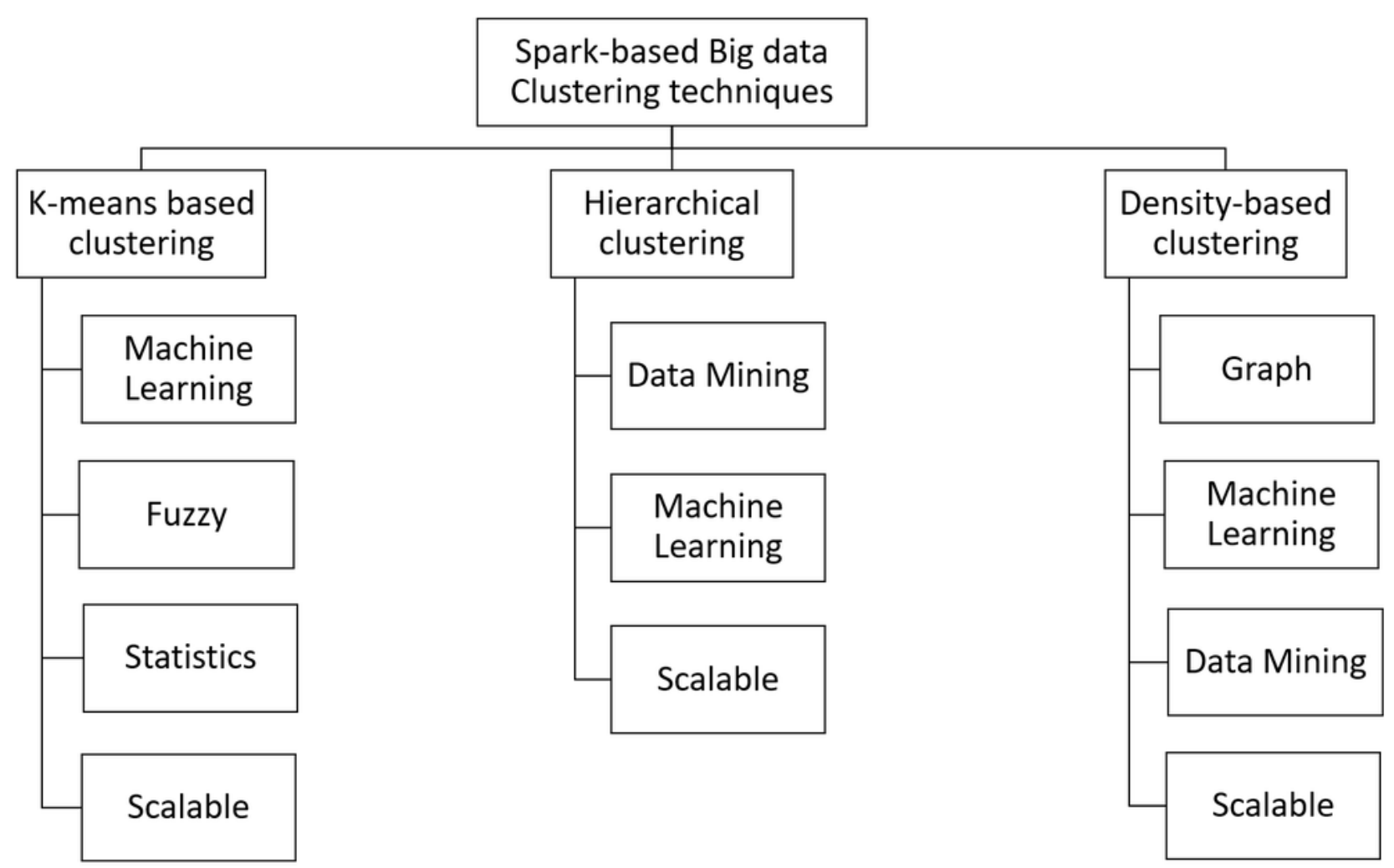




\section{Table $\mathbf{1}$ (on next page)}

Comparison of Spark-based Clustering methods in terms of the supported Big Data characteristic (volume, variety and velocity) and in terms of the type of data (real and synthetic) the proposed method was validated. 
2

3 Table 1: Comparison of Spark-based Clustering methods in terms of the supported Big Data

4 characteristic (volume, variety and velocity) and in terms of the type of data (real and synthetic) the

5 proposed method was validated.

\begin{tabular}{|c|c|c|c|c|c|c|c|}
\hline \multirow[t]{2}{*}{ Category } & \multirow{2}{*}{$\begin{array}{l}\text { Sub- } \\
\text { category }\end{array}$} & \multirow[t]{2}{*}{ Paper } & \multicolumn{3}{|c|}{$\begin{array}{c}\text { Supported Big Data } \\
\text { Characteristic }\end{array}$} & \multicolumn{2}{|c|}{ Validated on } \\
\hline & & & volume & variety & velocity & Real & Synthetic \\
\hline \multirow{28}{*}{ K-means } & \multirow{6}{*}{$\begin{array}{l}\text { Machine } \\
\text { Learning }\end{array}$} & [38] & $\sqrt{ }$ & & $\sqrt{ }$ & & $\sqrt{ }$ \\
\hline & & [41] & $\sqrt{ }$ & & & & $\sqrt{ }$ \\
\hline & & [47] & $\sqrt{ }$ & & & $\sqrt{ }$ & \\
\hline & & [60] & $\sqrt{ }$ & & & & $\sqrt{ }$ \\
\hline & & [62] & $\sqrt{ }$ & & & $\sqrt{ }$ & \\
\hline & & [63] & $\sqrt{ }$ & & $\sqrt{ }$ & $\sqrt{ }$ & $\sqrt{ }$ \\
\hline & \multirow{5}{*}{ Fuzzy } & [45] & & $\sqrt{ }$ & & $\sqrt{ }$ & \\
\hline & & [46] & $\sqrt{ }$ & & & $\sqrt{ }$ & \\
\hline & & [53] & $\sqrt{ }$ & & & $\sqrt{ }$ & \\
\hline & & [54] & $\sqrt{ }$ & & & $\sqrt{ }$ & \\
\hline & & [55] & $\sqrt{ }$ & & & $\sqrt{ }$ & \\
\hline & \multirow{4}{*}{ Statistics } & [50] & $\sqrt{ }$ & & & $\sqrt{ }$ & \\
\hline & & [57] & $\sqrt{ }$ & & & $\sqrt{ }$ & \\
\hline & & [58] & $\sqrt{ }$ & & & $\sqrt{ }$ & \\
\hline & & [61] & $\sqrt{ }$ & $\sqrt{ }$ & $\sqrt{ }$ & $\sqrt{ }$ & \\
\hline & \multirow{13}{*}{ Scalable } & [36] & $\sqrt{ }$ & $\sqrt{ }$ & & $\sqrt{ }$ & \\
\hline & & [37] & $\sqrt{ }$ & & & & $\sqrt{ }$ \\
\hline & & [39] & & $\sqrt{ }$ & $\sqrt{ }$ & $\sqrt{ }$ & \\
\hline & & [40] & $\sqrt{ }$ & & & $\sqrt{ }$ & \\
\hline & & [42] & $\sqrt{ }$ & & & $\sqrt{ }$ & \\
\hline & & [43] & $\sqrt{ }$ & $\sqrt{ }$ & & $\sqrt{ }$ & $\sqrt{ }$ \\
\hline & & [44] & $\sqrt{ }$ & $\sqrt{ }$ & & $\sqrt{ }$ & $\sqrt{ }$ \\
\hline & & [48] & $\sqrt{ }$ & & & $\sqrt{ }$ & $\sqrt{ }$ \\
\hline & & [49] & $\sqrt{ }$ & & & $\sqrt{ }$ & \\
\hline & & {$[51]$} & $\sqrt{ }$ & & & $\sqrt{ }$ & \\
\hline & & [52] & $\sqrt{ }$ & & & $\sqrt{ }$ & \\
\hline & & {$[56]$} & $\sqrt{ }$ & $\sqrt{ }$ & $\sqrt{ }$ & $\sqrt{ }$ & \\
\hline & & [59] & $\sqrt{ }$ & & & $\sqrt{ }$ & \\
\hline \multirow{4}{*}{ Hierarchical } & \multirow{3}{*}{$\begin{array}{c}\text { Data } \\
\text { Mining }\end{array}$} & [65] & $\sqrt{ }$ & & & $\sqrt{ }$ & \\
\hline & & [66] & $\sqrt{ }$ & & & & $\sqrt{ }$ \\
\hline & & [68] & $\sqrt{ }$ & & & & $\sqrt{ }$ \\
\hline & Machine & {$[70]$} & $\sqrt{ }$ & & & $\sqrt{ }$ & $\sqrt{ }$ \\
\hline
\end{tabular}




\begin{tabular}{|c|c|c|c|c|c|c|c|}
\hline & Learning & [71] & $\sqrt{ }$ & $\sqrt{ }$ & $\sqrt{ }$ & $\sqrt{ }$ & \\
\hline & \multirow{3}{*}{ Scalable } & [64] & $\sqrt{ }$ & & & $\sqrt{ }$ & \\
\hline & & [67] & $\sqrt{ }$ & $\sqrt{ }$ & $\sqrt{ }$ & $\sqrt{ }$ & \\
\hline & & [69] & & & & & $\sqrt{ }$ \\
\hline \multirow{14}{*}{ Density } & \multirow{4}{*}{ Graph } & [74] & $\sqrt{ }$ & & & $\sqrt{ }$ & \\
\hline & & [79] & $\sqrt{ }$ & & & $\sqrt{ }$ & $\sqrt{ }$ \\
\hline & & [80] & $\sqrt{ }$ & & & $\sqrt{ }$ & \\
\hline & & [82] & $\sqrt{ }$ & & & $\sqrt{ }$ & $\sqrt{ }$ \\
\hline & & [76] & $\sqrt{ }$ & & & & $\sqrt{ }$ \\
\hline & Data & [78] & $\sqrt{ }$ & & & $\sqrt{ }$ & $\sqrt{ }$ \\
\hline & & [83] & $\sqrt{ }$ & & & $\sqrt{ }$ & \\
\hline & & [72] & $\sqrt{ }$ & $\sqrt{ }$ & & $\sqrt{ }$ & \\
\hline & IVacnine & [77] & $\sqrt{ }$ & $\sqrt{ }$ & & $\sqrt{ }$ & $\sqrt{ }$ \\
\hline & & [84] & $\sqrt{ }$ & & & $\sqrt{ }$ & $\sqrt{ }$ \\
\hline & & [73] & $\sqrt{ }$ & & & $\sqrt{ }$ & \\
\hline & Scalable & [75] & $\sqrt{ }$ & & & $\sqrt{ }$ & $\sqrt{ }$ \\
\hline & scalable & [81] & $\sqrt{ }$ & & & & $\sqrt{ }$ \\
\hline & & [85] & & & $\sqrt{ }$ & $\sqrt{ }$ & \\
\hline
\end{tabular}




\section{Table 2 (on next page)}

Shows the data sources of the Spark-based clustering papers. 
1

2 Table 2: Shows the data sources of the Spark-based clustering papers.

3

\begin{tabular}{|c|c|}
\hline Data Source & Paper \\
\hline IEEE Explorer & $\begin{array}{l}{[2][4][5][7][9][18][19][25][27][36][37][38][39][40][41][43][45][48]} \\
{[49][53][54][56][61][62][64][65][67][68][69][70][73][75][83][84][86]} \\
{[88][90]}\end{array}$ \\
\hline Elsevier & {$[1][43][74]$} \\
\hline Springer & $\begin{array}{l}{[3][6][20][23][24][30][32][34][35][44][46][47][58][59][60][63][76]} \\
{[77][79][85][89]}\end{array}$ \\
\hline Google Scholar & [15] [42] [11] [12] [14] [26] [31] [57] [80] [82] [87] \\
\hline ResearchGate & [8] [13] [16] [17] [72] [33] [50] [51] [90] \\
\hline Science Direct & [10] [21] [22] [29] [52] [28] [66] [71] [74] [78] \\
\hline
\end{tabular}

4

5 


\section{Table 3 (on next page)}

Shows which papers in the survey were published in each of the last 6 years. 
2

3 Table 3: shows which papers in the survey were published in each of the last 6 years.

4

\begin{tabular}{|l|l|c|}
\hline $\begin{array}{c}\text { Year of } \\
\text { Publication }\end{array}$ & Papers & $\begin{array}{c}\text { Number } \\
\text { of Papers }\end{array}$ \\
\hline 2014 & {$[36][32][41][56][61][67][70][90]$} & 8 \\
\hline 2015 & {$[9][8][1][22][31][64][15][89]$} & 8 \\
\hline 2016 & $\begin{array}{l}\text { [1] [2] [7] [6] [11] [16] [28] [36] [37] [38] [39] [42] [48] [50] [55] [58] } \\
\text { [59] [65] [69] [79] [82] }\end{array}$ & 21 \\
\hline 2017 & {$[6][13][17][19][21][23][40][43][45][47][62][63][74][84][86]$} & 15 \\
\hline 2018 & $\begin{array}{l}\text { [3] [4] [15] [18] [20] [24] [26] [27] [29] [30] [44] [49] [60] [68] [73] } \\
\text { [75] [76] [80] [81] [83] [85] [88] }\end{array}$ & 22 \\
\hline 2019 & {$[12][46][51][52][53][54][57][72][77][78][87][91]$} & 12 \\
\hline 2020 & {$[66]$} & 1 \\
\hline
\end{tabular}

5

6

7 